\title{
Some new weighted compact embeddings results and existence of weak solutions for eigenvalue Robin problem
}

\author{
Ismail Aydin ${ }^{1}$ and Cihan UNAL ${ }^{2}$ \\ ${ }^{1}$ Sinop University \\ ${ }^{2}$ Assessment, Selection and Placement Center
}

November 9, 2020

\begin{abstract}
In this paper, we prove the existence and multiplicity of solutions for the following Robin problem $\backslash$ begin $\{$ equation* $\} \backslash$ left $\backslash\{$ $\backslash$ begin $\{$ array $\}\{$ cc $\}-\backslash$ text $\{\operatorname{div}\} \backslash \operatorname{left}\left(\mathrm{a}(\mathrm{x}) \backslash\right.$ left $\backslash$ vert $\backslash$ nabla $\mathrm{u} \backslash$ right $\backslash$ vert ${ }^{\wedge}\{\mathrm{p}(\mathrm{x})-2\} \backslash$ nabla $\mathrm{u} \backslash$ right $)=\backslash$ lambda $\mathrm{b}(\mathrm{x}) \backslash$ left $\backslash$ vert $\mathrm{u} \backslash$ right $\backslash$ vert ${ }^{\wedge}\{\mathrm{q}(\mathrm{x})-2\}, \& \mathrm{x} \backslash$ in $\backslash$ Omega $\backslash \backslash \mathrm{a}(\mathrm{x}) \backslash$ left $\backslash$ vert $\backslash$ nabla $\mathrm{u} \backslash$ right $\backslash$ vert ${ }^{\wedge}\{\mathrm{p}(\mathrm{x})-2\} \backslash$ frac $\{\backslash$ partial $\mathrm{u}\}\{\backslash$ partial $\backslash$ upsilon $\}+\backslash$ beta $(\mathrm{x}) \backslash$ left $\backslash$ vert $\mathrm{u} \backslash$ right $\backslash$ vert ${ }^{\wedge}\{\mathrm{p}(\mathrm{x})-2\} \mathrm{u}=0, \& \mathrm{x} \backslash$ in $\backslash$ partial $\backslash$ Omega,$\% \backslash$ end $\{$ array $\} \% \backslash$ right. \end } \text { equation* } \% \text { under some appropriate con- } ditions in double weighted variable exponent Sobolev space by applying Mountain Pass Lemma, Ekeland's variational principle and Fountain Theorem.
\end{abstract}

\section{Hosted file}

Aydin and Unal.pdf available at https://authorea.com/users/374347/articles/491884-somenew-weighted-compact-embeddings-results-and-existence-of-weak-solutions-for-eigenvaluerobin-problem 